\title{
Maternal Depression and Anxiety in Children and Adolescents with Intellectual Disability
}

\author{
Sundar Gnanavel ${ }^{1}$ \\ ${ }^{1}$ Child and Adolescent Mental Health Services, Tees, Esk and Wear \\ Valleys NHS foundation Trust, Newcastle Upon Tyne, United Kingdom
}

J Neurosci Rural Pract 2019;10:728-730

Anxiety and depression in mothers of children with intellectual disability poses a significant challenge in terms of clinical management and from a public health perspective. ${ }^{1}$ A recent meta-analysis demonstrated a higher level of depressive symptoms in mothers of children with developmental disabilities (effect size of 0.39) and 29\% of mothers of children with disabilities also had significantly elevated symptoms. ${ }^{2}$

It is widely believed that the different psychosocial stressors that are associated with being a mother of a child with intellectual disability possibly results in greater psychological morbidity, particularly anxiety and depressive symptoms. ${ }^{3}$ This relationship is truly bidirectional. It is to be remembered that maternal depression and anxiety can have a profound impact on a child's psychological, neurological, emotional, and social development. ${ }^{4}$ We also need to note that these parents might engage less with the child and adolescent services (e.g., parenting workshops) due to their psychological morbidity, resulting in poorer outcome for their children. The transactional relationship between child and parent behaviors is well documented and has definite implications in management of both these groups: mothers and children with intellectual disability. ${ }^{5}$

The methodological issues commonly encountered in these studies typically include use of only a screening questionnaire (rather than a clinical diagnosis of anxiety or depressive disorder), lack of distinction between symptoms and disorder in diagnostic terms, failure to consider alternative diagnosis such as adjustment disorder, use of inappropriate questionnaires/using different thresholds, and absence of a control group, for example, mother of children without any child with a disability. ${ }^{3}$ Many of these studies are cross-sectional in design and focus on a clinic attending sample rather than a community sample (limiting the generalizability of the findings).

The different maternal variables or correlates that are typically studied would include maternal intelligence quotient, maternal

\begin{abstract}
Address for correspondence Sundar Gnanavel, MD, MRCPsych, Child and Adolescent Mental Health Services, Tees, Esk and Wear Valleys NHS foundation Trust, 35 St. Anns Close, Newcastle Upon Tyne NE12QP, United Kingdom (e-mail: sundar221103@yahoo.com).
\end{abstract}

education, maternal physical health, socioeconomic status, family support, ethnicity, other stressors, maternal coping style, and several other children with disabilities in the family. The child variables that have been studied include severity of intellectual disability, presence of comorbidity, and other service-related variables such as access to health/social services. ${ }^{6}$

It is also interesting to note the possibility of a biological link between gene mutations associated with intellectual disability and vulnerability to anxiety/depression. For example, premutation carrier mothers of those children with intellectual disability associated with fragile $\mathrm{X}$ syndrome possibly are more prone to mood and anxiety disorders due to the carrier status. ${ }^{7}$ This is likely to open new avenues for research around specific treatment modalities for these mothers.

The future direction of research should possibly incorporate gold standard assessment tools for identifying mood/anxiety disorders, utilize a longitudinal approach with multiple points of assessment, and include the confounding effect of any treatment availed, for example, psychotherapy/psychotropic medication. While there is a plethora of research around association and correlates of different variables with maternal depression/ anxiety in this context, there is very little empirical evidence around bespoke approaches to manage this psychological morbidity in mothers, particularly from developing resource stretched countries.

It is highly likely that a holistic model including biological vulnerability, poor support system, poor coping strategies, and lack of appropriate services might explain maternal anxiety/ depression in this context. Identifying methods of identifying and managing psychological morbidity early in these mothers would go a long way in addressing the cumulative psychological morbidity of this special group of children and their mothers. This would ideally involve an active liaison between adult, child, and perinatal mental health services (where available).

\section{Conflict of Interest}

None declared. 


\section{References}

1 Karande S, Kumbhare N, Kulkarni M, Shah N. Anxiety levels in mothers of children with specific learning disability. J Postgrad Med 2009;55(3):165-170

2 Singer GH. Meta-analysis of comparative studies of depression in mothers of children with and without developmental disabilities. Am J Ment Retard 2006;111(3):155-169

3 Bailey DB, Jr, Golden RN, Roberts J, Ford A. Maternal depression and developmental disability: research critique. Ment Retard Dev Disabil Res Rev 2007;13(4):321-329

4 Dawson G, Ashman SB, Panagiotides H, et al. Preschool outcomes of children of depressed mothers: role of maternal behavior, contextual risk, and children's brain activity. Child Dev 2003;74(4):1158-1175

5 Alvarez SL, Meltzer-Brody S, Mandel M, Beeber L. Maternal depression and early intervention: a call for an integration of services. Infants Young Child 2015;28(1):72-87

6 Carpiniello B, Piras A, Pariante CM, Carta MG, Rudas N. Psychiatric morbidity and family burden among parents of disabled children. Psychiatr Serv 1995;46(9):940-942

7 Hagerman RJ, Hagerman PJ. The fragile X premutation: into the phenotypic fold. Curr Opin Genet Dev 2002;12(3):278-283 